\title{
ON PIERCE-LIKE IDEMPOTENTS AND HOPF INVARIANTS
}

\author{
GIORA DULA and PETER HILTON
}

Received 3 March 2003

\begin{abstract}
Given a set $K$ with cardinality $\|K\|=n$, a wedge decomposition of a space $Y$ indexed by $K$, and a cogroup $A$, the homotopy group $G=[A, Y]$ is shown, by using Pierce-like idempotents, to have a direct sum decomposition indexed by $P(K)-\{\phi\}$ which is strictly functorial if $G$ is abelian. Given a class $\rho: X \rightarrow Y$, there is a Hopf invariant $\mathrm{HI}_{\rho}$ on $[A, Y]$ which extends Hopf's definition when $\rho$ is a comultiplication. Then $\mathrm{HI}=\mathrm{HI}_{\rho}$ is a functorial sum of $\mathrm{HI}_{L}$ over $L \subset K,\|L\| \geq 2$. Each $\mathrm{HI}_{L}$ is a functorial composition of four functors, the first depending only on $A^{n+1}$, the second only on $d$, the third only on $\rho$, and the fourth only on $Y^{n}$. There is a connection here with Selick and Walker's work, and with the Hilton matrix calculus, as described by Bokor (1991).
\end{abstract}

2000 Mathematics Subject Classification: 55Q25, 55P30, 55P45.

1. Introduction. In an earlier paper [5], to which this may be regarded as a sequel, the authors introduced a definition of a Hopf invariant which generalized most (but not all) existing definitions. We recall that definition.

Working in the pointed homotopy category $\mathscr{H}$, we consider a cogroup $A$ in $\mathscr{H}$ with comultiplication $\mu: A \rightarrow A \vee A$. We now suppose given a map $d: A \rightarrow X$ and a map $\rho: X \rightarrow Y_{1} \vee Y_{2}$. If the projections of $\rho$ onto $Y_{1}$ and $Y_{2}$ are $\alpha_{1}$ and $\alpha_{2}$, respectively, so that $\alpha_{i}: X \rightarrow Y_{i}$, then $\rho$ is referred to as a copairing of $\alpha_{1}$ and $\alpha_{2}$, which are themselves described as copairable (see [5]).

We have a diagram

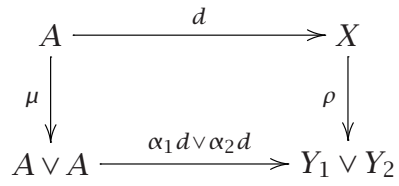

which does not, in general, commute. However, we may embed (1.1) in the larger diagram

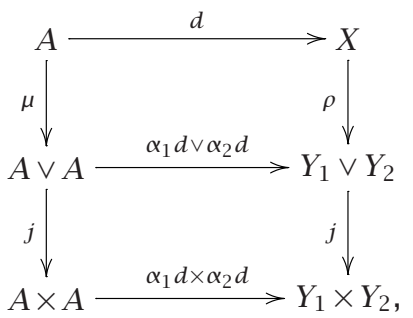


where $j$ is the canonical map; and, in (1.2), the bottom square and the composite square both commute. That the composite square commutes follows from the relations $j \mu=\Delta: A \rightarrow A \times A$ and $j \rho=\left\{\alpha_{1}, \alpha_{2}\right\}: X \rightarrow Y_{1} \times Y_{2}$.

Let $Y_{1} b Y_{2}$ be the homotopy fiber of $j: Y_{1} \vee Y_{2} \rightarrow Y_{1} \times Y_{2}$. Then it follows from what we have said that $\rho d-\left(\alpha_{1} d \vee \alpha_{2} d\right) \mu$ lifts to $Y_{1} b Y_{2}$. Moreover, analysis of $j: Y_{1} \vee Y_{2} \rightarrow Y_{1} \times Y_{2}$ shows that the lift is unique. We call this lift, as an element of $\left[A, Y_{1} b Y_{2}\right]$, the Hopf invariant of $d$ with respect to $\rho$, and write it as $\mathrm{HI}_{\rho}(d)$.

In this paper, we pursue the study of $\mathrm{HI}_{\rho}$, but allow ourselves one further freedom. Instead of starting with a copairing $\rho$, which may be thought of as a kind of a counion of two maps, we consider a counion of $n$ maps, which we call an $n$-counion map $\rho: X \rightarrow Y=Y_{1} \vee Y_{2} \vee \cdots \vee Y_{n}$. The rest of the definition of $\mathrm{HI}_{\rho}(d)$ will be essentially the same, except that, for simplicity, we confine our attention to the case in which $A$ is a commutative cogroup. Since our principal interest in defining Hopf invariants in $\mathscr{H}$ would be in the case in which $A$ is, at least, a double suspension, we argue that this gain in simplicity is obtained at relatively low cost.

With $[A, Y]$ commutative-indeed, we write it additively, as is customary with the higher homotopy groups - we may bring to bear the notion of orthogonal idempotents on $[A, Y]$. Thus let $\pi_{i}: Y \rightarrow Y_{i}, \iota_{i}: Y_{i} \rightarrow Y$ be the canonical projection onto the $i$ th summand in $Y$ and the corresponding injection. Then $\pi_{i} \iota_{i}$ is the identity on $Y_{i}$ and $e_{i}=\iota_{i} \pi_{i}: Y \rightarrow Y$ is an idempotent map. Moreover,

$$
e_{i} e_{j}=0, \quad i \neq j
$$

Then $e_{i}$ induces an idempotent endomorphism of $[A, Y]$ which we also call $e_{i}$; and if $i \neq j$, then $e_{i}$ and $e_{j}$ are orthogonal idempotent endomorphisms.

On the other hand, it is not true that $e_{1}+e_{2}+\cdots+e_{n}=1$. Indeed, if $f: A \rightarrow Y$, the mapping $f \mapsto f_{0}=f-\sum_{i=1}^{n} e_{i} f$ is an endomorphism of $[A, Y]$ and, in fact, is itself idempotent. For

$$
e_{i} f_{0}=e_{i} f-\sum_{i=1}^{n} e_{j} e_{i} f=e_{j} f-e_{j} f=0
$$

so $f_{0}-\sum_{i=1}^{n} e_{i} f_{0}=f_{0}$. We call this idempotent $e_{0}$. Then $e_{0} e_{j}=e_{j}-\sum_{i=1}^{n} e_{i} e_{j}=0$ and $e_{j} e_{0}=0$, as shown above. Thus $e_{0}, e_{1}, \ldots, e_{n}$ form a complete Pierce-like system of orthogonal idempotents on $[A, Y]$, and we may say that it is the nontriviality of $e_{0}$ which allows us, or requires us, to define a Hopf invariant. Notice that the commutativity of $[A, Y]$ has greatly facilitated this last discussion.

In Section 2, we define the Hopf invariant of $d: A \rightarrow X$ with respect to the $n$-counion map $\rho: X \rightarrow Y$ as the lift of $e_{0}(\rho d)$ to the homotopy fiber of the canonical map $j: Y \rightarrow Y_{1} \times Y_{2} \times \cdots \times Y_{n}$. We show in what sense the Hopf invariant-which is a homomorphism of commutative groups-is natural and we analyse it as a sum of $2^{n}-n-1$ elements, each factoring through a given space determined by a summand $Y_{i_{1}} \vee Y_{i_{2}} \vee \cdots \vee Y_{i_{k}}$ of $Y$ with $k \geq 2$. Notice 
that such an analysis is vacuous in the case $n=2$. We also relate our Hopf invariant to the one given in [4] in connection with the calculation of relative attaching maps for Thom spaces.

In Section 3, we make an entirely different analysis of the Hopf invariant, representing it as the composition of four maps, each depending on some aspect of the original data. One of the constituent maps appears to be closely related to a very general kind of Hopf invariant defined by Walker [11].

The constructions and arguments in this paper are all carried out in the pointed homotopy category $\mathscr{H}$. However, they may be couched in categorytheoretic language and executed, with minor modifications, in a general category possessing pullbacks, coproducts, and zero object (see [5]). In particular, we might, as in [5], study these ideas in the category of groups. We might also consider the dual concepts. Indeed, the dual concept is related to a paper of Selick [10].

In Section 4, we show the relationship of the idempotents of this paper, of span 2, to the matrix calculus introduced in [7] and exploited in [2].

Since we always work on $\mathscr{H}$, we do not regard it as always necessary to mention the base point explicitly nor to insist on distinguishing notationally between a map and its homotopy class.

2. The Hopf invariant and naturality. Let the space $Y$ be given as a wedge (coproduct)

$$
Y=Y_{1} \vee Y_{2} \vee \cdots \vee Y_{n}
$$

We may describe $Y$ as a costructured space, with summands $Y_{i}$. Thus we associate with $Y$ the projections

$$
\pi_{i}: Y \longrightarrow Y_{i}
$$

and injections

$$
\iota_{i}: Y_{i} \rightarrow Y
$$

Then $\pi_{i} \iota_{i}=\operatorname{Id}_{i}: Y_{i} \rightarrow Y_{i}$ and $e_{i}=\iota_{i} \pi_{i}: Y \rightarrow Y$ is an idempotent map, $i=$ $1,2, \ldots, n$. We say that the idempotents $e_{i}$ are associated with the costructure on $Y$. Notice that these idempotents are orthogonal, that is, $e_{i} e_{j}=0, i \neq j$.

Let $A$ be a commutative cogroup in $\mathscr{H}$ (e.g., a double suspension). Then $[A, Y]$ is a commutative group and the idempotents $e_{i}$ induce an orthogonal system of idempotent endomorphisms, which we also denote by $e_{i}$,

$$
e_{i}:[A, Y] \longrightarrow[A, Y]
$$

In general, the system $\left\{e_{i}\right\}$ of idempotents on $[A, Y]$ is not complete; for example, if $A=S^{3}$ and $Y=S^{2} \vee S^{2}$, then there is a Whitehead product element $\left[\operatorname{Id}_{1}, \operatorname{Id}_{2}\right] \in \pi_{3}\left(S^{2} \vee S^{2}\right)$ of infinite order, not expressible as $e_{1} \alpha_{1}+e_{2} \alpha_{2}$. 
However, for any $f \in[A, Y]$, we set

$$
f_{0}=f-\sum_{i=1}^{n} e_{i} f \in[A, Y] .
$$

Proposition 2.1. The function $f \mapsto f_{0}$ is an idempotent endomorphism $e_{0}$ of $[A, Y]$. Moreover, the system $\left\{e_{0}, e_{1}, \ldots, e_{n}\right\}$ is a complete system of orthogonal idempotents on $[A, Y]$.

Proof. Write $f_{0}=e_{0} f$. Then $e_{0}(f+g)=f+g-\sum_{i=1}^{n} e_{i}(f+g)=f+g-$ $\sum_{i=1}^{n}\left(e_{i} f+e_{i} g\right)=\left(f-\sum_{i=1}^{n} e_{i} f\right)+\left(g-\sum_{i=1}^{n} e_{i} g\right)=e_{0} f+e_{0} g$. Thus $e_{0}$ is an endomorphism. Also

$e_{0} f_{0}=f_{0}-\sum_{i=1}^{n} e_{i} f_{0}=f_{0}, \quad e_{j} f_{0}=e_{j} f-\sum_{i=1}^{n} e_{j} e_{i} f=e_{j} f-e_{j} f=0, \quad j=1,2, \ldots, n$.

Thus, $e_{0} f_{0}=f_{0}$, so $e_{0}$ is idempotent; and, by the argument above, $e_{j} e_{0}=0$, $j=1,2, \ldots, n$. The formal calculation

$$
e_{j} e_{0}=e_{j}\left(1-e_{1}-\cdots-e_{n}\right)=e_{j}-e_{j} e_{1}-\cdots-e_{j} e_{n}=e_{j}-e_{j}=0
$$

would indeed have sufficed, and it shows equally well that $e_{0} e_{j}=0$. We conclude that $\left\{e_{0}, e_{1}, \ldots, e_{n}\right\}$ constitutes a system of orthogonal idempotents on $[A, Y]$ such that $e_{0}+e_{1}+\cdots+e_{n}=1$, as was to be proved.

Now let $j$ be the natural inclusion

$$
j: Y=Y_{1} \vee Y_{2} \vee \cdots \vee Y_{n} \rightarrow \prod Y=Y_{1} \times Y_{2} \times \cdots \times Y_{n}
$$

and let $b(Y)$ be the homotopy fiber of $j$. For any $f \in[A, Y]$, consider the diagram

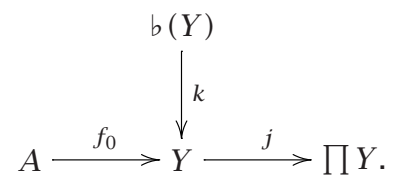

Now $j=\left\{\pi_{1}, \pi_{2}, \ldots, \pi_{n}\right\}$, so $j f_{0}=\left\{\pi_{1} f_{0}, \pi_{2} f_{0}, \ldots, \pi_{n} f_{0}\right\}$. Moreover,

$$
\pi_{s} e_{i}= \begin{cases}0, & i \neq s, \\ \pi_{s}, & i=s,\end{cases}
$$

so that $\pi_{s} f_{0}=\pi_{s}\left(1-e_{1}-\cdots-e_{n}\right) f=\left(\pi_{s}-\pi_{s}\right) f=0$. Hence $j f_{0}=0$ and $f_{0}$ lifts into $b(Y)$.

LEMMA 2.2. The lift of $f_{0}$ into $b(Y)$ is unique. 
Proof. We have the fiber sequence

$$
\Omega(b Y) \rightarrow \Omega Y \rightarrow \Omega \prod Y \rightarrow b Y \rightarrow Y \rightarrow \prod Y
$$

inducing the exact sequence

$$
[A, \Omega Y] \stackrel{j_{*}}{\longrightarrow}\left[A, \Omega \prod Y\right] \longrightarrow[A, b Y] \stackrel{k_{*}}{\longrightarrow}[A, Y] \stackrel{j_{*}}{\longrightarrow}\left[A, \prod Y\right]
$$

Now $[A, \Pi Y]=\bigoplus_{i}\left[A, Y_{i}\right]$ and $j_{*}$ obviously maps $[A, Y]$ onto $\left[A, Y_{i}\right]$. Thus $j_{*}:[A, Y] \rightarrow[A, \Pi Y]$ is surjective. Equally well $j_{*}:[A, \Omega Y] \rightarrow\left[A, \Omega \prod Y\right]$ is surjective, so $k_{*}:[A, b Y] \rightarrow[A, Y]$ is injective.

REMARK 2.3. Clearly $j_{*}:[A, Y] \rightarrow[A, \Pi Y]$ has a right inverse $m$, given by

$$
m \mid\left[A, Y_{i}\right]=\iota_{i *} .
$$

Thus

$$
[A, b Y] \stackrel{k_{*}}{\longrightarrow}[A, Y] \stackrel{j_{*}}{\longrightarrow}\left[A, \prod Y\right]
$$

is a split short exact sequence. Notice that the validity of this remark depends on the commutativity of $A$; so does much of the preceding reasoning.

We write $\ell(f)$ for the lift of $f_{0}$ into $b(Y)$. Since $e_{0}$ and $k_{*}$ are homomorphisms, it follows that

$$
\ell:[A, Y] \longrightarrow[A, b(Y)]
$$

is a homomorphism.

We now analyse $\ell(f)$. Let $Y^{\prime}=Y_{i_{1}} \vee \cdots \vee Y_{i_{k}}, 1 \leq i_{1}<i_{2}<\cdots<i_{k} \leq n$. We call $Y^{\prime}$ a summand or simply a subspace of the costructured space $Y$ of span $k$ and write $\left|Y^{\prime}\right|=k$. We plainly have maps

$$
\pi: Y \rightarrow Y^{\prime}, \quad \iota: Y^{\prime} \rightarrow Y, \quad \pi: \prod Y \rightarrow \prod Y^{\prime}, \quad \iota: \prod Y^{\prime} \rightarrow \prod Y
$$

and inducing maps

$$
\pi_{Y^{\prime}}: b Y \rightarrow b Y^{\prime}, \quad \iota_{Y^{\prime}}: b Y^{\prime} \longrightarrow b Y
$$

such that

$$
\pi_{Y^{\prime}} \iota_{Y^{\prime}}=\mathrm{Id}, \quad \iota_{Y^{\prime}} \pi_{Y^{\prime}}=e_{Y^{\prime}}: b Y \rightarrow b Y,
$$

with $e_{Y^{\prime}}$ an idempotent map.

In particular, suppose that $\left|Y^{\prime}\right|=2$. Then, as $Y^{\prime}$ ranges over the subspaces of $Y$ of span 2, we obtain a system of orthogonal idempotents $e_{Y^{\prime}}$ on $b Y$. We also obtain a map

$$
\pi: b Y \rightarrow \prod_{\left|Y^{\prime}\right|=2} b Y^{\prime}
$$


with components $\pi_{Y^{\prime}}$. Thus (compare the definition of $\ell(f)$ above) for any map $\ell: A \rightarrow b Y$, we may lift

$$
\ell-\sum_{\left|Y^{\prime}\right|=2} e_{Y^{\prime}} \ell
$$

to the homotopy fiber of $\pi$ (2.19), which we call $b^{2} Y$. Moreover, the lift is unique so that

$$
\ell=\sum_{\left|Y^{\prime}\right|=2} e_{Y^{\prime}} \ell+k^{(2)} \ell^{(2)}
$$

where $\ell^{(2)}$ is the lift of $\ell-\sum_{\left|Y^{\prime}\right|=2} e_{Y^{\prime}} \ell$ so that

$$
A \stackrel{\ell^{(2)}}{\longrightarrow} b^{2} Y \stackrel{k^{(2)}}{\longrightarrow} b Y
$$

is a fiber sequence. Note that $e_{Y^{\prime}} \ell$ factors through $b Y^{\prime}$.

We now continue the process of analysing $\ell$. We next have to analyse $\ell^{(2)}$. We now allow $Y^{\prime}$ to range over all subspaces of $Y$ of span 3 . We thus obtain maps

$$
\pi_{Y^{\prime}}: b^{2} Y \longrightarrow b^{2} Y^{\prime}, \quad \iota_{Y^{\prime}}: b^{2} Y^{\prime} \longrightarrow b^{2} Y
$$

such that

$$
\pi_{Y^{\prime}} \iota_{Y^{\prime}}=\mathrm{Id}, \quad \iota_{Y^{\prime}} \pi_{Y^{\prime}}=e_{Y^{\prime}}: b^{2} Y \longrightarrow b^{2} Y
$$

with $e_{Y^{\prime}}$ an idempotent map. As before, we obtain a map

$$
\pi: b^{2} Y \rightarrow \prod_{\left|Y^{\prime}\right|=3} b^{2} Y^{\prime}
$$

with components $\pi_{Y^{\prime}}$. The maps $e_{Y^{\prime}}$ on $b^{2} Y$ constitute a system of orthogonal idempotents so that the map $\ell^{(2)}: A \rightarrow b^{2} Y$ may be written as

$$
\ell^{(2)}=\sum_{\left|Y^{\prime}\right|=3} e_{Y^{\prime}} \ell^{(2)}+k^{(3)}+\ell^{(3)}
$$

where $\ell^{(3)}$ is the lift of $\ell^{(2)}-\sum_{\left|Y^{\prime}\right|=3} e_{Y^{\prime}} \ell^{(2)}$ to $b^{3} Y$, the homotopy fiber of $\pi$ of (2.25).

The process terminates when we arrive at the set of subspaces of span ( $n-$ 1 ). We will then write $\ell$ as a sum of $2^{n}-n-1$ maps, corresponding to the subspaces of $Y$ of span greater that or equal to 2 (including $Y$ itself). Thus, we have proved the following proposition. 
Proposition 2.4. The set $\left\{e_{Y^{\prime}}\left|Y^{\prime} \subset Y,\right| Y^{\prime} \mid \geq 2\right\}$ is a complete system of $2^{n}-n-1$ orthogonal idempotents on the group $[A, b Y]$ even though $b Y$ is not given as a wedge of spaces. As a corollary, any map $\ell: A \rightarrow b Y$ may be uniquely expressed as the sum of $2^{n}-n-1$ terms $\ell_{Y^{\prime}}$, each factoring through a subspace $b^{k-1} Y^{\prime}$ of $b Y$ that corresponds to a subspace $Y^{\prime}$ of $Y$ of span $k \geq 2$.

Notice that the mystery of the number $2^{n}-n-1$ is dissolved if one observes the corollary or the equivalent statement.

THEOREM 2.5. The set $\left\{k e_{Y^{\prime}} \ell\left|Y^{\prime} \subset Y,\right| Y^{\prime} \mid \geq 1\right\}$ is a complete system of $2^{n}-1$ orthogonal idempotents on the group $[A, Y]$, obtained from the fact that $Y$ is a wedge of $n$ subspaces. Thus any map $f: A \rightarrow Y$ may be uniquely expressed as the sum of $2^{n}-1$ terms $f_{Y^{\prime}}$, each factoring through a subspace $b^{k-1} Y^{\prime}$ of $Y$ that corresponds to a subspace $Y^{\prime}$ of $Y$ of span $k \geq 1$.

REMARK 2.6. In Theorem 2.5 , the idempotent $k e_{Y^{\prime}} \ell$ simply means $e_{Y^{\prime}}$ if $\left|Y^{\prime}\right|=1$.

We next discuss the naturality of $\ell$. Let $g: A \rightarrow B$ be a homomorphism of commutative cogroups and let $Y=Y_{1} \vee Y_{2} \vee \cdots \vee Y_{n}$ and $Z=Z_{1} \vee Z_{2} \vee \cdots \vee Z_{n}$ be two costructured spaces. Finally, let $h: Y \rightarrow Z$ be a costructure-preserving map, that is, $h\left(Y_{i}\right) \subseteq Z_{i}, i=1,2, \ldots, n$. We then prove

THEOREM 2.7. If the diagram

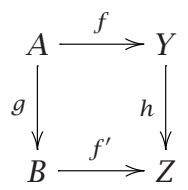

commutes, then so does the diagram

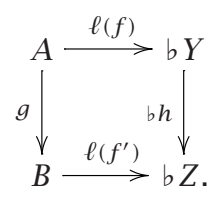

Proof. We first remark that $b$ is obviously a functor $b: \mathscr{H}^{n} \rightarrow \mathscr{H}$, so $b h$ is defined. Now, to prove the commutativity of (2.28), we first consider the diagram

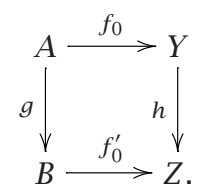


Then $h f_{0}=h\left(f-\sum_{i \geq 1} e_{i} f\right)=h f-\sum_{i \geq 1} h e_{i} f$. But $h e_{i}=h \iota_{i} \pi_{i}=\iota_{i} h_{i} \pi_{i}=$ $\iota_{i} \pi_{i} h=e_{i} h$, where $h_{i}: Y_{i} \rightarrow Z_{i}$ is obtained by restricting $h$. Thus

$$
\begin{aligned}
h f_{0} & =h f-\sum e_{i} h f \\
& =f^{\prime} g-\sum e_{i} f^{\prime} g \\
& =\left(f^{\prime}-\sum e_{i} f^{\prime}\right) g, \quad \text { since } g \text { is a homomorphism } \\
& =f_{0}^{\prime} g,
\end{aligned}
$$

establishing the commutativity of (2.29).

Now we have an obvious commutative diagram (used, in fact, to present $b$ as a functor)

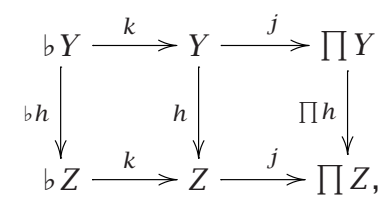

so the commutativity of (2.28) follows from (2.29) and (2.31), using the fact that $k$ is a monomorphism.

We may further refine the naturality as follows. Let $Y^{\prime}$ be a summand of $Y$ of span $k \geq 2$ and let $\ell_{Y^{\prime}}(f)$ be the corresponding component of $\ell(f)$, regarded as a map $\ell_{Y^{\prime}}(f): A \rightarrow b^{k-1} Y^{\prime}$. Let $Z^{\prime}$ be the corresponding summand of $Z$ and define $\ell_{Z^{\prime}}\left(f^{\prime}\right): B \rightarrow b^{k-1} Z^{\prime}$ similarly. Then $h$ induces $b^{k-1} h: b^{k-1} Y^{\prime} \rightarrow b^{k-1} Z^{\prime}$ and the diagram

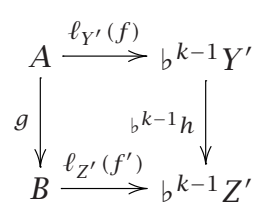

commutes. We leave the details to the reader, remarking that we may reexpress this refinement by asserting the naturality of the idempotents and summation described in Proposition 2.4.

We now formally introduce the Hopf invariant. A map $\rho: X \rightarrow Y$ is called an $n$-counion map or, more precisely, an $n$-counion of the maps $\pi_{i} \rho: X \rightarrow Y_{i}$, $i=1,2, \ldots, n$. If $n=2$, this is called a copairing (see [5]). Now $\rho$ induces a homomorphism $\rho:[A, X] \rightarrow[A, Y]$ and, for $d: A \rightarrow X$, we define the Hopf invariant of $d$, relative to $\rho$, to be $\ell(\rho d) \in[A, b Y]$. Plainly this defines a homomorphism

$$
\mathrm{HI}=\mathrm{HI}_{\rho}:[A, X] \longrightarrow[A, b Y] .
$$

We may now invoke Proposition 2.4, applied to the map $\ell=\ell(\rho d)=\mathrm{HI}_{\rho}(d)$, to conclude 
THEOREM 2.8. The Hopf invariant $\mathrm{HI}_{\rho}:[A, X] \rightarrow[A, b Y]$ may be expressed as the sum of $2^{n}-n-1$ homomorphisms $\mathrm{HI}_{Y^{\prime}}$, each factoring through a group $\left[A, b^{k-1} Y^{\prime}\right]$ corresponding to a subspace $Y^{\prime}$ of $Y$ of span $k \geq 2$.

We say that the Hopf invariant is thereby expressed as a sum of constituent subinvariants.

The naturality of the Hopf invariant now expresses itself as follows. We suppose a given commutative diagram

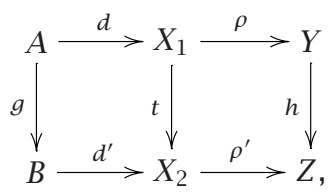

where $g$ is a homomorphism of commutative cogroups and $h$ is costructurepreserving. Then (2.33) induces a commutative diagram

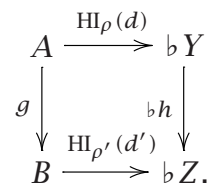

There is also a refined form of this naturality statement, based on (2.32), involving the constituent subinvariants. It is further of interest to interpret the vanishing of the Hopf invariant; thus

THEOREM 2.9. The vanishing of $\mathrm{HI}_{\rho}(d)$ is equivalent to the assertion that

$$
\rho d=e_{1} \rho d+e_{2} \rho d+\cdots+e_{n} \rho d
$$

Proof. $\ell(\rho d)=0 \Leftrightarrow(\rho d)_{0}=0 \Leftrightarrow \rho d-e_{1} \rho d-e_{2} \rho d-\cdots-e_{n} \rho d=0$. Notice that $e_{i} \rho d=\iota_{1} \alpha_{i} d$, where $\rho$ is a counion of $\alpha_{1}, \alpha_{2}, \ldots, \alpha_{n}$, with $\alpha_{i}: X \rightarrow Y_{i}$.

A version of Theorems 2.5, 2.7, 2.8, and 2.9 appeared in [3].

The above definition of the Hopf invariant generalizes that given in the case $n=2$ in [5], where it is further related to a number of existing definitions. Staying always with the case $n=2$, but confining attention to coactions (or cooperations, see [6]) $\rho$, there is a treatment in [4] which is specialized to a stage in the version of Hilton and Milnor $[1,8]$ when the coaction is a comultiplication.

EXAMPLE 2.10. Let $P_{1}, P_{2}, \ldots, P_{n}$ be spaces and let $T^{(1)}=T^{(1)}\left(P_{1}, P_{2}, \ldots, P_{n}\right)$ be the fat wedge of $\sum P_{1}, \sum P_{2}, \ldots, \sum P_{n}$, in the terminology of Ganea. Thus $T^{(1)}$ is the subspaces of $\sum P_{1} \times \sum P_{2} \times \ldots \times \sum P_{n}$ consisting of points with at least 
one coordinate at the base point. There is then a relative homeomorphism

$$
C\left(P_{1} * P_{2} * \cdots * P_{n}\right), P_{1} * P_{2} * \cdots * P_{n} \longrightarrow \sum P_{1} \times \sum P_{2} \times \cdots \times \sum P_{n}, T^{(1)},
$$

where $P_{1} * P_{2} * \cdots * P_{n}$ is the (iterated) join. Let $w_{n}$ be the associated attaching map, that is,

$$
w_{n}: P_{1} * P_{2} * \cdots * P_{n} \longrightarrow T^{(1)}
$$

is the restriction of the map (2.37).

In the same way, we may consider, for each $Q_{j}=P_{1} * P_{2} * \cdots * \hat{P}_{j} * \cdots * P_{n}$, the attaching map

$$
w_{n-1, j}: Q_{j} \longrightarrow T^{(1)}\left(P_{1}, P_{2}, \ldots, \hat{P}_{j}, \ldots, P_{n}\right), \quad j=1,2, \ldots, n
$$

Now the union of the spaces $T^{(1)}\left(P_{1}, P_{2}, \ldots, \hat{P}_{j}, \ldots, P_{n}\right)$ is $T^{(2)}\left(P_{1}, P_{2}, \ldots, P_{n}\right)$ "the next fattest wedge," that is, the subspace of $\sum P_{1} \times \sum P_{2} \times \cdots \times \sum P_{n}$ consisting of points with at least two coordinates at the base point. Moreover, the maps $w_{n-1, j}$ combine to produce a map

$$
w_{n-1}: Q_{1} \vee Q_{2} \vee \cdots \vee Q_{n} \longrightarrow T^{(2)}
$$

whose mapping cone is precisely $T^{(1)}$.

In general, given a map $f: A \rightarrow B$ with mapping cone $C_{f}$, there is, as explained in [6], a cooperation or coaction $\rho: C_{f} \rightarrow \sum A \vee C_{f}$ of $\sum A$ on $C_{f}$. In our case, with $f=w_{n-1}$ in (2.40), $\rho$ becomes a map

$$
\rho: T^{(1)} \longrightarrow \sum Q_{1} \vee \sum Q_{2} \vee \cdots \vee \sum Q_{n} \vee T^{(1)}
$$

and thus an $(n+1)$-counion map. The map (2.41) may be fed into our definition to produce essentially the Hopf invariant of [4] and to motivate our definition in this paper.

3. A canonical factorization of the Hopf invariant. In this section we describe a canonical expression for $\mathrm{HI}_{\rho}(d)$ as a composition of four maps, each depending on a particular ingredient of the definition of the Hopf invariant.

Given $f: A \rightarrow Y=Y_{1} \vee Y_{2} \vee \cdots \vee Y_{n}$, where $A$ is a commutative cogroup, we may express $f_{0}$ as the composition

$$
A \stackrel{\mu^{(n+1)}}{\longrightarrow} \bigvee_{n+1} A \stackrel{\left\langle f,-e_{1} f, \ldots,-e_{n} f\right\rangle}{\longrightarrow} Y,
$$


where $\mu: A \rightarrow A \vee A$ is the comultiplication. Further factorizing the right-hand map in (3.1) and slightly modifying the relevant factors yield the composition

$$
A \stackrel{\sigma}{\longrightarrow} \bigvee_{n+1} A \stackrel{\vee f}{\longrightarrow} \bigvee_{n+1} Y \stackrel{\left\langle 1, e_{1}, \ldots, e_{n}\right\rangle}{\longrightarrow} Y
$$

of $f_{0}$, where $\sigma$ is the sum of the identity 1, mapping $A$ to the first summand in $\bigvee_{n+1} A$, and maps -1 , mapping $A$ into the second, third, $\ldots,(n+1)$ th summand of $\bigvee_{n+1} A$. We could write $\sigma=1_{1}-1_{2}-\cdots-1_{n+1}$.

Now let $j$, as before, be the canonical map from $\bigvee_{i} B_{i}$ to $\prod_{i} B_{i}$. We may then embed (3.2) in the commutative diagram

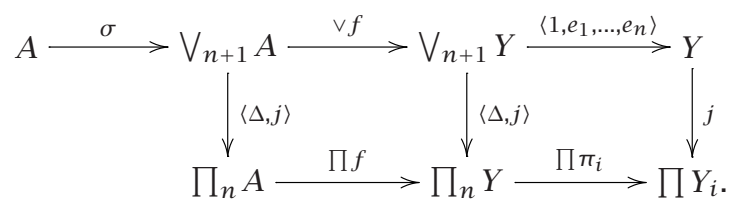

Here $\Delta: A \rightarrow \prod A$ and $\Delta: Y \rightarrow \prod Y$ are diagonal maps. The commutativity of the left-hand square in (3.3) is obvious. As for the right-hand square, we observe that $\left(\prod \pi_{i}\right) \Delta y_{k}=\left(*, \ldots, *, y_{k}, *, \ldots, *\right)=j y_{k}$ if $y_{k} \in Y_{k}$; and that, if $y \in(m+1)$ th copy of $Y$ in $\bigvee_{n+1} Y, m \geq 1$, then

$$
\begin{aligned}
\left(\prod \pi_{i}\right) j y & =\prod \pi_{i}(*, \ldots, *, y, *, \ldots, *), \\
& =\left(*, \ldots, *, \pi_{m} y, *, \ldots, *\right) \\
& =j e_{m} y,
\end{aligned}
$$

where $y$ appears in the $m$ th factor $Y$ in $\prod_{n} Y$. Further, it is clear that $\langle\Delta, j\rangle \sigma=$ $0: A \rightarrow \prod_{n} A$ for $j\left(1_{2}+1_{3}+\cdots+1_{n+1}\right)=\Delta$. Thus $\sigma$ lifts to $\kappa: A \rightarrow F_{A}$, where $F_{A}$ is the homotopy fiber of $\langle\Delta, j\rangle$, and we may embed (3.3) in the commutative diagram

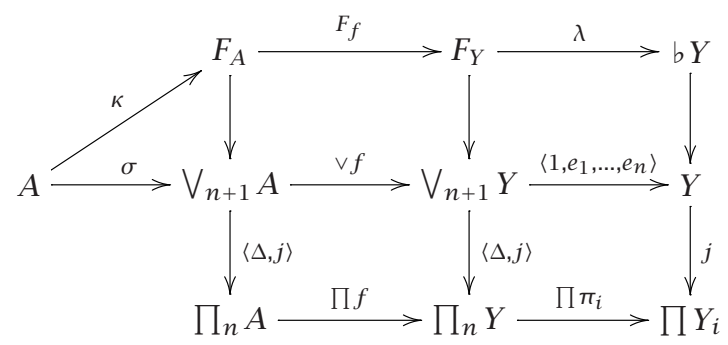

and obviously

$$
\lambda\left(F_{f}\right) \kappa=\ell(f)
$$


We now revert to the Hopf invariant. We only have to replace $f$ in (3.5) by $f=\rho d$,

$$
A \stackrel{d}{\longrightarrow} X \stackrel{\rho}{\longrightarrow} Y .
$$

Thus it is a matter of factoring the $\left(F_{f}, \vee f, \Pi f\right)$-column of (3.5) in the obvious way to obtain

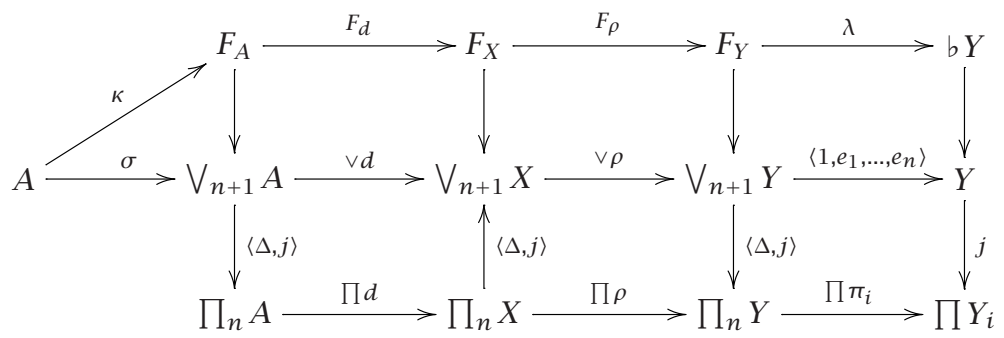

and the factorization

$$
\mathrm{HI}_{\rho}(d)=\lambda(F \rho)\left(F_{d}\right) \kappa
$$

This is the canonical factorization of the title of this section.

If the counion of size $n$ is assumed to be given, then we notice that, in (3.9),

(i) $\kappa$ depends only on $A$;

(ii) $F_{d}$ depends only on $d$;

(iii) $F_{\rho}$ depends only on $\rho$;

(iv) $\lambda$ depends only on $Y$, with its costructure.

Without going into details, we make the obvious remark that a similar factorization is available for each of the $2^{n}-n-1$ constituent subinvariants of the Hopf invariant in the sense of Theorem 2.8.

REMARKS. (i) The factor $F_{\rho}$ of the Hopf invariant $\mathrm{HI}_{\rho}(d)$ in (3.9) is related to Walker's version of the Hopf invariant [11]. Walker starts with a pair of maps

$$
B \stackrel{f}{\longrightarrow} A \stackrel{g}{\longrightarrow} C
$$

and constructs the double mapping cylinder $Z=Z_{f, g}$. There is thus a commutative square

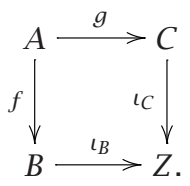


Let $P$ be the homotopy pullback of $i_{B}$ and $i_{C}$ in (3.11), creating a commutative diagram

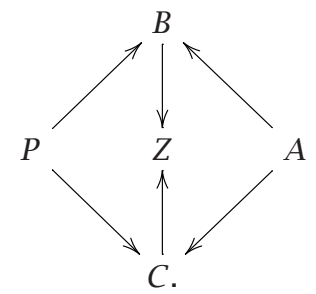

We now pinch $A$ to a point in (3.11). We think of $B$ and $C$ in (3.11) as having been replaced by the mapping cylinders of $f$ and $g$, respectively, so that (3.11) becomes a strict pushout of inclusion maps. When we pinch $A$ to a point throughout (3.11), the mapping cylinders become mapping cones $C_{f}$ and $C_{g}$ and $Z$ becomes the one-point union (coproduct) $C_{f} \vee C_{g}$. Thus (3.11) turns into the diagram

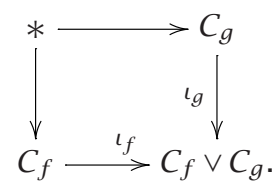

Following Walker, we designate the homotopy pullback of $\iota_{f}$ and $\iota_{g}$ by $C_{f} \hat{*}$ $C_{g}$. We thus obtain the diagram

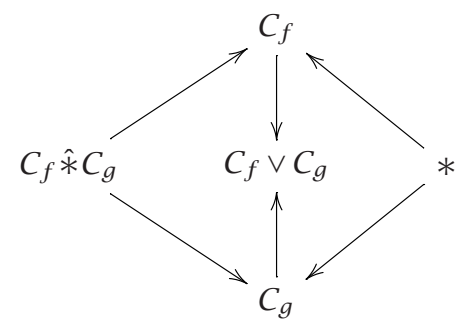

and the pinching maps induce a map of diagram (3.12) to diagram (3.14). The component

$$
P \longrightarrow C_{f} \hat{*} C_{g}
$$

of this map of the diagrams is Walker's Hopf invariant. If we write $\rho$ for the component

$$
\rho: Z \longrightarrow C_{f} \vee C_{g}
$$

of this map of diagrams, then this $\rho$ is a two-counion map and plays the same role as in our definition of the Hopf invariant. 
We conjecture that Walker's Hopf invariant is closely related to the map $F_{\rho}$ of (3.8), with $X=Z$ and $Y=C_{f} \vee C_{g}$.

(ii) It is plain that the maps $\kappa$ of the factorization (3.9) for various values of $n$ may be related. We write $\kappa_{n}$ and $\sigma_{n}$ for the maps $\kappa$ and $\sigma$ of (3.8). Then $\sigma_{1}=1_{1}-1_{2}: A \rightarrow A \vee A$ and there are maps

$$
q=\sigma_{1} \vee 1_{3} \vee \cdots \vee 1_{n+2}: \bigvee A \longrightarrow \bigvee_{n+2} A, \quad r: \bigvee_{n+2} A \longrightarrow \bigvee_{n+1} A
$$

where $r$ projects off the second summand $A$. Then

$$
r q=1: \bigvee_{n+1} A \longrightarrow \bigvee_{n+1} A, \quad q \sigma_{n}=\sigma_{n+1}: A \longrightarrow \bigvee_{n+2} A
$$

Now let $\bar{q}: \prod_{n} A \rightarrow \prod_{n+1} A$ map $\underline{a}$ to $(*, \underline{a})$ and let $\bar{r}: \prod_{n+1} A \rightarrow \prod_{n} A$ project off the first component. Then the diagram

$$
\begin{gathered}
\bigvee_{n+1} A \underset{r}{\stackrel{q}{\rightleftarrows}} \bigvee_{n+2} A \\
\downarrow\langle\Delta, j\rangle \\
\prod_{n} A \underset{\bar{q}}{\stackrel{\bar{q}}{\rightleftarrows}} \prod_{n+1} A
\end{gathered}
$$

commutes so that there are induced maps, which we again write as $q, r$, thus

$$
F_{A, n} \underset{r}{\stackrel{q}{\gtrless}} F_{A, n+1}
$$

such that

$$
r q=1: F_{A, n} \longrightarrow F_{A, n}, \quad q \kappa_{n}=\kappa_{n+1}: A \longrightarrow F_{A, n+1} .
$$

We may use the maps $q$ to pass to the limit $F_{A \infty}$, obtaining a map $\kappa_{\infty}: A \rightarrow F_{A \infty}$ which is independent of $n$ and thus truly universal.

(iii) There is a relation between the duals of the constructions in this paper and some aspects of Selick's paper [10]. In [10, Section 3, page 408], Selick's D is a dual for the present Hopf invariant based on two summands for the particular case of a multiplication. Selick's $D_{n}$ is a sum of duals of the Hopf invariant associated to $e_{Y^{\prime}},\left|Y^{\prime}\right| \geq 2$, in the case of a multiplication. Selick's $f_{i}$ is the dual of our $e_{Y^{\prime}} f$ for the case $\left|Y^{\prime}\right|=i$ and multiplication. In [10, Lemma 1, page 408], he proves that the $n$-fold multiplication of $f$ is a sum of $2^{n}-1$ summands. In the proof of [10, Lemma 5, page 410], he starts with the summands of [10, Lemma 1] and then moves the $n$ summands that correspond to $e_{Y^{\prime}},\left|Y^{\prime}\right|=1$, to the side of $f$, getting on the right-hand side a sum of $2^{n}-n-1$ summands that correspond to $e_{Y^{\prime}},\left|Y^{\prime}\right| \geq 2$, mirroring our result in Theorem 2.8. 
4. The Hilton quadratic matrix calculus. The quadratic matrix calculus defined in [7] and used in [2, 7, 9] can be obtained by using the present paper idempotents of span 2. We here use [2] as a source and deduce some of the results of [2, Section 4].

Let $\alpha: S^{4 m-1}=A=X \rightarrow S^{2 m} \vee S^{2 m} \vee \cdots \vee S^{2 m}=\bigvee_{K} S^{2 m}=Y$ be given as on [2, page 373]. Then $\alpha$ is a $K$-counion map as defined in the introduction and thus induces idempotents $\left\{e_{Y^{\prime}},\left|Y^{\prime}\right| \geq 2\right\}$ on the group $\left[S^{4 m-1}, b Y\right]$.

It can be observed that for $\left|Y^{\prime}\right|=2, b Y^{\prime}$ is the fiber of the map $S^{2 m} \vee S^{2 m} \rightarrow$ $S^{2 m} \times S^{2 m}$, which, as in [4], is equivalent to $\Omega\left(S^{2 m}\right) * \Omega\left(S^{2 m}\right)$, which, by James' celebrated formula [8], equals $S^{4 m-1} \cup e^{6 m-2} \cup \cdots$. Thus $\left[S^{4 m-1}, b Y^{\prime}\right]=\left[S^{4 m-1}\right.$, $\left.S^{4 m-1}\right]=\mathscr{L}$.

Also, for $\left|Y^{\prime}\right|=3$, say $Y^{\prime}=\{a, b, c\}, b Y^{\prime}$ can be embedded in the following commutative diagram:

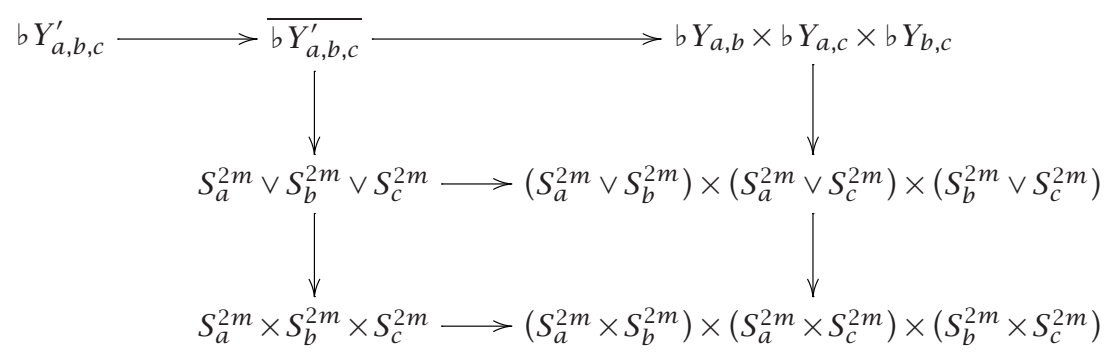

and by the well-known Hilton-Milnor Theorem, the first cell in $b Y_{a, b, c}$ is $\left(S_{a}^{2 m-1} *\right.$ $\left.S_{b}^{2 m-1}\right) \wedge S_{c}^{2 m-1}$ which is $(6 m-3)$-connected so that $\left[A, b Y_{a, b, c}^{\prime}\right]=0$. Thus the only idempotents in Theorem 2.5 correspond to span less than three. In particular, on $[A, Y]$, we have

$$
1=\sum_{i=1}^{K} e_{i}+\sum_{1 \leq i<j \leq K} e_{i, j},
$$

and in particular

$$
f=\sum_{i=1}^{K} f_{i}+\sum_{1 \leq i<j \leq K} f_{i, j},
$$

where $f_{i, j}$ factors as

$$
S^{4 m-1} \longrightarrow b Y_{i, j} \stackrel{\simeq}{\longrightarrow} S^{4 m-1} \stackrel{w_{2}}{\longrightarrow} S_{i}^{2 m} \vee S_{j}^{2 m} \stackrel{\text { inc }}{\longrightarrow} Y .
$$

Thus $f_{i, j}$ is determined completely by the degree of the map $S^{4 m-1} \rightarrow S^{4 m-1}$, denoted by $a_{i j}$ on [2, page 373]. 
For $i=j, f_{i, j}=f_{j}$ factors as

$$
A=S^{4 m-1} \stackrel{f}{\longrightarrow} Y=\bigvee_{K} S_{i}^{2 m} \stackrel{\pi_{j}}{\longrightarrow} S_{j}^{2 m} \stackrel{\mathrm{inc}_{j}}{\longrightarrow} Y .
$$

Now $\pi_{j} f: S^{4 m-1} \rightarrow S^{2 m}$ has a Hilton-Milnor invariant obtained by considering $\rho f: S^{4 m-1} \rightarrow S^{2 m} \vee S^{2 m}$, where $\rho$ is the comultiplication map. The invariant of $f$ is a map $a_{i, i}: S^{4 m-1} \rightarrow S^{4 m-1}$. Thus the following matrix is obtained:

$$
\underline{H}(f)= \begin{cases}a_{i j}=a_{j i}, & i \neq j, \\ a_{i i}, & i=j,\end{cases}
$$

defined on [2, page 373] and called the Hilton-Hopf quadratic form of $f$.

Given any map $f: \bigvee_{J} S^{4 m-1} \rightarrow \bigvee_{K} S^{2 m}$, it is determined by the inclusions $f_{j}: S^{4 m-1} \stackrel{\mathrm{in}_{j}}{\longrightarrow} \bigvee_{J} S^{4 m-1} \stackrel{f}{\rightarrow} \bigvee_{K} S^{2 m}$, each of which is determined by a $K \times K$ integer matrix as above. Then $f$ is determined by $J$ integer matrices which can be written using juxtaposition as a single $K \times J K$ integer matrix composed of $J$ blocks:

$$
\underline{H}(f)=\left(\underline{H}\left(f_{1}\right), \underline{H}\left(f_{2}\right), \ldots, \underline{H}\left(f_{J}\right)\right)
$$

as described on [2, page 375].

Finally, for a cogroup $A$ and $Y=\bigvee_{K} S^{m}$, any map $\phi: A \rightarrow Y$ has a decomposition as above: $\phi=\sum_{\left|Y^{\prime}\right| \geq 1} e_{Y^{\prime}} \phi$. If $A=Y=\bigvee_{K} S^{m}$, then every $e_{Y^{\prime}},\left|Y^{\prime}\right|>1$, factors through a multiconnected space and is thus null. Thus we have $\phi=$ $\sum_{j=1}^{K} \phi_{j}$, where $\phi_{j}$ is a map $Y \rightarrow Y \stackrel{\pi_{j}}{\longrightarrow} S_{j}^{m} \hookrightarrow Y$. Each map out of a wedge is determined by the restrictions $S_{j}^{m} \hookrightarrow Y \stackrel{\phi_{j}}{\longrightarrow} S_{j}^{m}$ so that $\phi: Y \rightarrow Y$ is determined by $K \times K$ maps $S_{i}^{m} \stackrel{\mathrm{in}_{i}}{\hookrightarrow} Y \stackrel{\phi}{\rightarrow} Y \stackrel{\pi_{j}}{\longrightarrow} S_{j}^{m}$, which are determined by degrees. Thus $\phi=\left\{\operatorname{deg}\left(\phi_{j, i}\right)\right\}=\underline{A}(\phi)$ is a $K \times K$ matrix, defined exactly as in [2, Lemma 4.5(iii)]. (The reader may find, in what follows, some superficial changes of notation from that of [2].) We now describe the matrix calculus.

Suppose the following given composition:

$$
\underset{J}{\bigvee} S^{4 m-1} \stackrel{\psi}{\longrightarrow} \bigvee_{J} S^{4 m-1} \stackrel{f}{\longrightarrow} \bigvee_{K} S^{2 m} \stackrel{\phi}{\longrightarrow} \bigvee_{K} S^{2 m}
$$

Then $\psi$ is determined by a $J \times J$ integer degree matrix $\underline{A}(\psi), \phi$ by a $K \times K$ integer degree matrix $\underline{A}(\phi)$, and $f$ leads to an integer $K \times J K$ matrix of the Hilton quadratic form $\underline{H}(f)$. Also $\phi f \psi$ creates an integer $K \times J K$ matrix of the quadratic form $\underline{H}(\phi f \psi)$. Then, for $i<j$, the $(i, j)$ th term of the matrix $\underline{H}(\phi f \psi)$ is given by using the projection $\pi_{i, j} \phi f \psi: \bigvee_{K} S^{4 m-1} \rightarrow S_{i}^{2 m} \vee S_{j}^{2 m}$ and a lifting $\bigvee_{J} S^{4 m-1} \rightarrow S^{4 m-1} \stackrel{w_{i, j}}{\longrightarrow} S_{i}^{2 m} \vee S_{j}^{2 m}$. 
Map (4.8) can be presented using inclusions in the following way:

$$
\operatorname{com}_{i, j}^{k}: S_{k}^{4 m-1} \stackrel{\mathrm{inc}_{k}}{\longrightarrow} \bigvee_{J} S^{4 m-1} \stackrel{\phi f \psi}{\longrightarrow} \bigvee_{K} S^{4 m-1} \stackrel{\pi_{i, j}}{\longrightarrow} S_{i}^{2 m} \vee S_{j}^{2 m}
$$

for $1 \leq i<j \leq K$ and $1 \leq k \leq J$.

Thus there are $K \times J K$ terms as above. Then we have the composition

$$
\operatorname{com}_{i, j}^{k}: S_{k}^{4 m-1} \stackrel{\mathrm{inc}_{k}}{\longrightarrow} \bigvee_{J} S^{4 m-1} \stackrel{\psi}{\longrightarrow} \bigvee_{J} S^{4 m-1} \stackrel{f}{\longrightarrow} \bigvee_{K} S^{2 m} \stackrel{\phi}{\longrightarrow} \bigvee_{K} S^{2 m} \stackrel{\pi_{i, j}}{\rightarrow} S_{i}^{2 m} \vee S_{j}^{2 m}
$$

This last map equals

$$
\pi_{i, j} \phi f \psi \text { inc }_{k}=\pi_{i, j} \phi\left(\sum_{1 \leq p<q \leq K} e_{p, q}+\sum_{s=1}^{K} e_{s}\right) f\left(\sum_{r=1}^{J} e r\right) \psi \text { inc }_{k} .
$$

As $e_{s}$ factors through $S^{2 m}$ and is null into $S^{4 m-1}$, then $\operatorname{com}_{i, j}^{k}$ equals

$$
\sum_{1 \leq p<q \leq K, 1 \leq r \leq J} \pi_{i, j} \phi e_{p, q} f e_{r} \psi \text { inc }_{k} .
$$

Any of the summands is a composition of three maps:

$$
\begin{gathered}
\pi_{r} \psi \text { inc }_{k}: S_{k}^{4 m-1} \stackrel{\mathrm{inc}_{k}}{\longrightarrow} \bigvee_{J} S^{4 m-1} \stackrel{\psi}{\longrightarrow} \bigvee_{J} S^{4 m-1} \stackrel{\pi_{r}}{\longrightarrow} S_{r}^{4 m-1}, \\
\pi_{p, q} f \text { inc }_{r}: S_{r}^{4 m-1} \stackrel{\mathrm{inc}_{r}}{\longrightarrow} \bigvee_{J} S^{4 m-1} \stackrel{f}{\longrightarrow} \bigvee_{K} S^{2 m} \stackrel{\pi_{p, q}}{\longrightarrow} S_{p}^{2 m} \vee S_{q}^{2 m}, \\
\pi_{i, j} \phi \text { inc }_{p, q}: S_{p}^{2 m} \vee S_{q}^{2 m} \stackrel{\text { inc } p, q^{\longrightarrow}}{\bigvee} S_{K} S^{2 m} \stackrel{\phi}{\longrightarrow} \bigvee_{K} S^{2 m} \stackrel{\pi_{i, j}}{\longrightarrow} S_{i}^{2 m} \vee S_{j}^{2 m} .
\end{gathered}
$$

The map in (4.13) is by definition $\underline{A}(\psi)_{r}^{k}$. The second part (4.14) defines a lifting $S_{r}^{4 m-1} \rightarrow S_{p, q}^{4 m-1}$ which is, by definition, $\underline{H}(f)_{p, q}^{r}$; and the map of the fibers $S_{p, q}^{4 m-1} \rightarrow S_{i, j}^{4 m-1}$ obtained from (4.14) is induced by applying the matrix $\bar{\phi}_{i, j}^{p, q}$, which is a $2 \times 2$ submatrix of $\underline{A}(\phi)$.

Thus we get the formula

$$
\underline{H}(\phi f \psi)_{i, j}^{k}=\underline{A}(\phi)_{i, j}^{p, q} \underline{H}(f)_{p, q}^{r} \underline{A}(\psi)_{r}^{k} .
$$

Recall that $\underline{A}(\phi)_{i, j}^{p, q}=\underline{A}(\phi)_{i}^{p} \otimes \underline{A}(\phi)_{j}^{q}$. Now we consider the matrices as written in [2].

Thus $\underline{H}(f)_{p, q}^{r}$ is written in the $(p,(r-1) K+q)$ th spot. While $\underline{H}(f)_{i, j}^{k}$ is written in the $(i,(k-1) K+j)$ th spot. The way to express the indices of matrices so as to accord with the multiplication is

$$
(i, p)(p,(r-1) K+q)((r-1) K+q,(k-1) K+j) .
$$

The correct matrix setup for this is $\underline{A}(\phi) \underline{H}(f)\left(\underline{A}(\psi) \otimes \underline{A}(\alpha)^{t}\right)$, which is the first line of [2, Lemma 4.8]. 
ACKNOWLEDGMENT. The first author would like to thank Imi Bokor for his hospitality during July 1999 and for many helpful discussions concerning [2], which led to the material of Section 4.

\section{REFERENCES}

[1] I. Berstein and P. J. Hilton, On suspensions and comultiplications, Topology 2 (1963), 73-82.

[2] I. Bokor, On genus and cancellation in homotopy, Israel J. Math. 73 (1991), no. 3, 361-379.

[3] G. Dula, Idempotents, universal arrows for Hopf invariant, and the Hopf invariant defined by Walker, preprint, 1991.

[4] _ On conic spaces, Algebraic Topology Poznań 1989 (S. Jackowski, B. Oliver, and K. Pawał owski, eds.), Lecture Notes in Math., vol. 1474, Springer, Berlin, 1991, pp. 38-58.

[5] G. Dula and P. J. Hilton, On pairable and copairable morphisms, Algebra Colloq. 1 (1994), no. 2, 128-138.

[6] B. Eckmann and P. J. Hilton, Operators and cooperators in homotopy theory, Math. Ann. 141 (1960), 1-21.

[7] P. J. Hilton, On the Grothendieck group of compact polyhedra, Fund. Math. 61 (1967), 199-214.

[8] J. W. Milnor, On the Construction FK, Mimeographed Lecture Notes, Princeton University, New Jersey, 1956.

[9] E. A. Molnar, Relations between wedge cancellation and localization for complexes with two cells, J. Pure Appl. Algebra 3 (1973), 141-158.

[10] P. Selick, Odd primary torsion in $\pi_{k}\left(S^{3}\right)$, Topology 17 (1978), no. 4, 407-412.

[11] M. Walker, Homotopy pullbacks and the Hopf invariant, J. London Math. Soc. (2) 19 (1979), no. 1, 153-158.

Giora Dula: Netanya College, P.O. Box 120, Neot Ganim, Netanya 42365, Israel

Peter Hilton: Department of mathematical Sciences, State University of New York at Binghamton, NY 13902-6000, USA

E-mail address: marge@math. binghamton.edu 


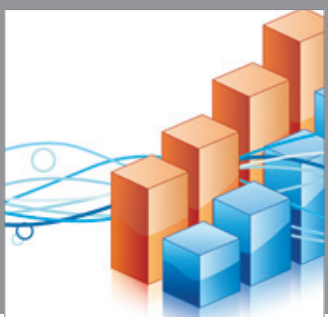

Advances in

Operations Research

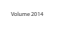

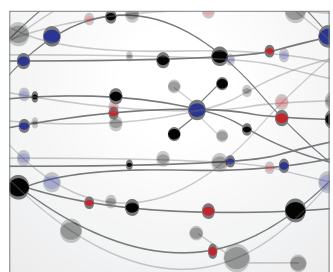

\section{The Scientific} World Journal
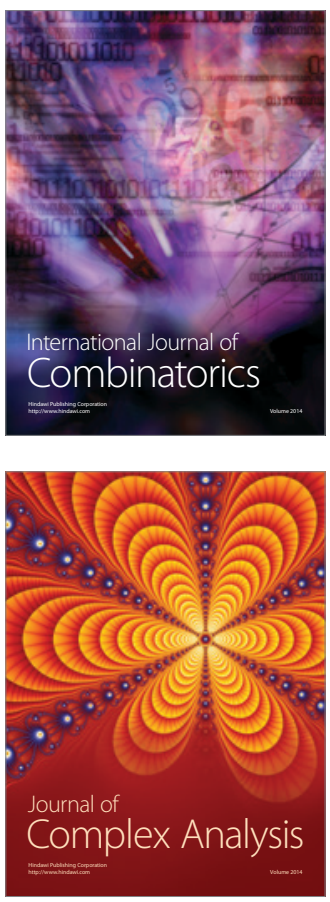

International Journal of

Mathematics and

Mathematical

Sciences
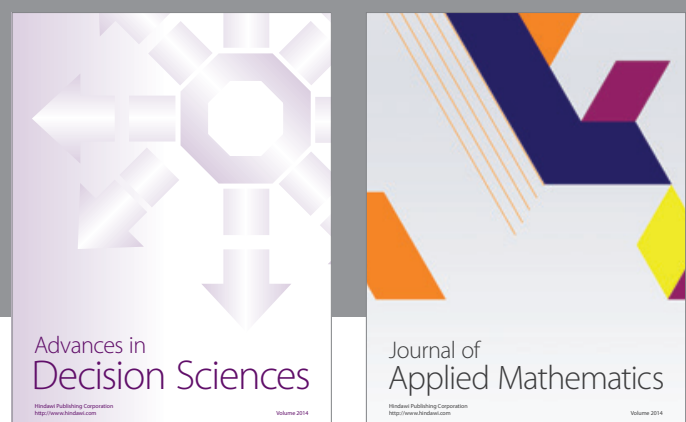

Journal of

Applied Mathematics
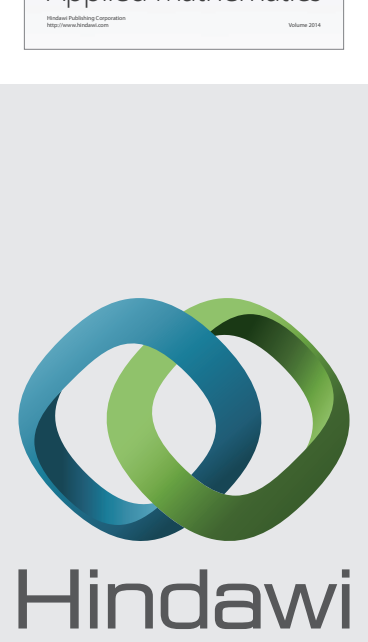

Submit your manuscripts at http://www.hindawi.com
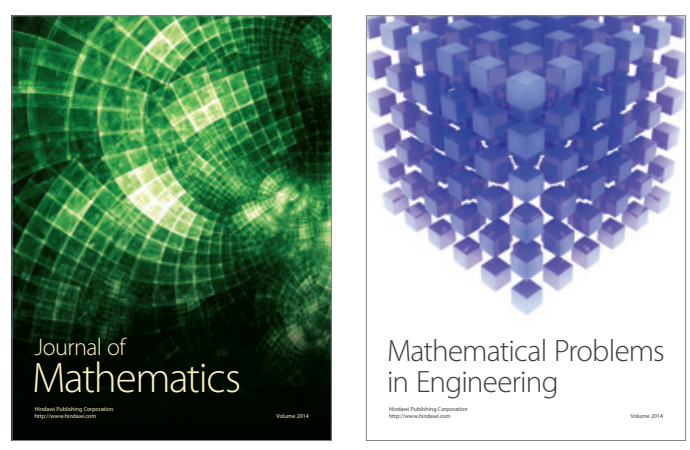

Mathematical Problems in Engineering
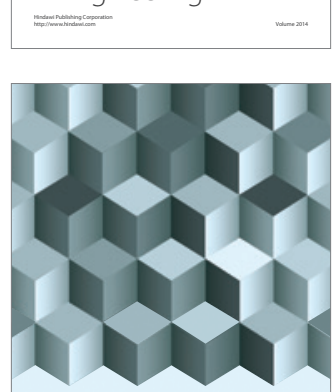

Journal of

Function Spaces
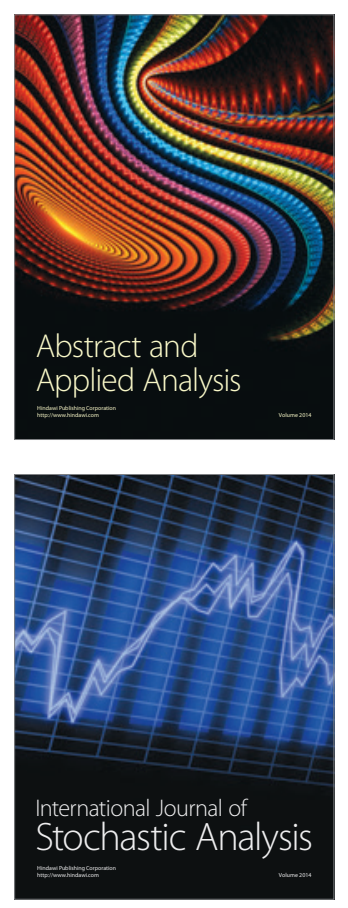

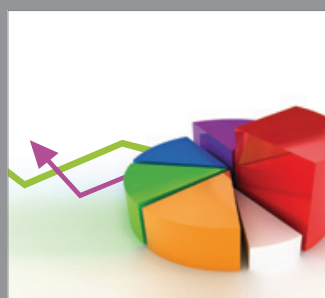

ournal of

Probability and Statistics

Promensencen
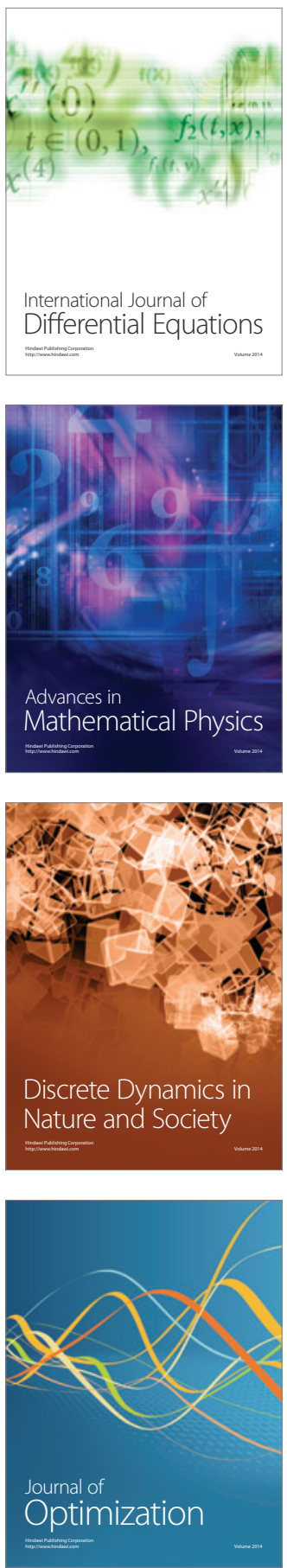